\title{
Dietary and Lifestyle Modifications among Diabetic Patients at a Tertiary Care Hospital in Delhi: A Cross-sectional Study
}

\author{
Gurmeet Kaur ${ }^{1}$, Priya Arora ${ }^{2}$, Jyotismita Pathak ${ }^{3}$, Ashok Maan ${ }^{4}$, Garima Monga ${ }^{5}$, Rajesh Vaidya ${ }^{6}$, Abhimanyu Kumar ${ }^{7}$
}

\begin{abstract}
Background: Modern lifestyle changes combined with urbanization has led to increased prevalence of diabetes. To decrease the morbidity, mortality, and the complications of diabetes, comprehension of diet and physical exercise is a must.

Objectives: To assess the degree of awareness of diet, physical exercise, and lifestyle modifications among type 2 diabetics among the urban population of Delhi.

Materials and methods: The study was conducted using a pretested questionnaire among 100 type 2 diabetic patients attending the medicine outpatient department of a Tertiary Care Hospital, Delhi. Baseline parameters of the study participants were expounded and their awareness and practice regarding the lifestyle modifications were assessed.

Results: The mean age of the study population was found to be $50 \pm 9.64$. An estimated $74 \%$ were well aware of foods to be eluded; $54 \%$ were familiar with the quantity of food in food plate. The awareness of lifestyle modifications was good among $29 \%$ and practice was followed by $15 \%$ of diabetic patients.

Conclusion: Despite the clear attitudes of type 2 diabetes patients towards dietary pattern and healthy lifestyle modifications, the lifestyle awareness and practices were poor among the study group.

Keywords: Awareness, Diabetes, Dietary Habits, Lifestyle modifications, Physical activity, Urbanization.

Journal of Medical Academics (2019): 10.5005/jp-journals-10070-0031
\end{abstract}

\section{INTRODUCTION}

India has more than 62 million diabetic patients in India, ${ }^{1,2}$ and projected to 79.4 million by the year $2030 .{ }^{1}$ A research steered by the Indian Council of Medical Research (ICMR) detailed that a partial population is affected in states of Northern India (Chandigarh 0.12 million, Jharkhand 0.96 million) as equated to Maharashtra (9.2 million) and Tamil Nadu (4.8 million). ${ }^{1,3}$ "Diabetes is a metabolic disease defined by hyperglycemia owing to defects in insulin secretion, insulin action, or both." ${ }^{\prime 4}$ The persistent hyperglycemia in diabetes affects all the body organs, including eyes, kidneys, nerves, heart, and blood vessels. It is cumulative arrays and reduced physical activity levels combined with the genetic predisposition, possibly the main causes pouring the current diabetes epidemic in the South Asian region. ${ }^{5}$ In North India, people mainly consume wheat and rice as a staple diet. ${ }^{6}$ Owing to several sociocultural concerns, protein ingestion arrays diverges among population amid vegetarians and non-vegetarians. Although the conservative lifestyle comprises robust physical activity followed by food rich in high fiber, whole-grain-based diet, vegetables, and fruits. The urban lifestyle comprising physical inactivity is recognized to be linked with obesity, diabetes, hypertension, and metabolic syndrome. ${ }^{7}$ The American Diabetes Association advocates 30 minutes of moderate-to-vigorous power aerobic exercise at least 5 days a week or a total of 150 minutes/week, and some style of muscle training at least 2 times/week in addition to aerobic activity. ${ }^{8,9}$ Revolution toward a facility sector economy and mounting use of advanced technologies have guided to a rapid waning in physical activity.

Various studies have inferred that diminished physical activity was observed amongst South Asians when equated to other ethnic groups. ${ }^{10,11}$ Diabetes is polygenic disease; therefore, knowledge of diabetes and its risk factors and lifestyle modifications aid in the control of disease. Nevertheless, the understanding is still insufficient about the true magnitude of disease among the diabetics. Although
${ }^{1-6}$ Department of Community Medicine, Army College of Medical
Sciences, Delhi, India
${ }^{7}$ Department of Orthopaedics, Vardhman Mahavir Medical College and Safdarjung Hospital, New Delhi, India

Corresponding Author: Priya Arora, Department of Community Medicine, Army College of Medical Sciences, Delhi, India, Phone: +91 8588806900, e-mail: drpriarora@rediffmail.com

How to cite this article: Kaur G, Arora P, Pathak J, et al. Dietary and Lifestyle Modifications among Diabetic Patients at a Tertiary Care Hospital in Delhi: A Cross-sectional Study. J Med Acad 2019;2(1):20-24.

Source of support: Nil

Conflict of interest: None

a plenty of literature reveals the prevalence of diabetes, only a handful of research focuses on the knowledge and practices of dietary intake, food perceptions, and physical activity. Most of the population in India has poor health-seeking behavior and is not accustomed to practice healthcare until the illness gets exaggerated, which contributes to the intensified morbidity status in the country.

Diabetes mellitus (DM) is an incorrigible but manageable disease. Self-discipline over physical exercise is an imperative contributor to boost the insulin sensitivity. Understanding about the caloric requirements, dietary pattern, and physical exercise is needed to fight against DM and to achieve normal glycemic control. Hence, this research brings in an insight on food practices, dietary perceptions, and lifestyle modifications to assess the levels of awareness of diet, physical exercise, and lifestyle modifications among type 2 diabetic patients from Tertiary Care Hospital of Delhi.

\section{Materials and Methods}

This cross-sectional study was conducted at the Tertiary Care Hospital located in an urban area, Delhi. This study assessed the 
awareness and practices about diet, physical exercise, and health education among type 2 DM patients. This research includes 100 diabetics attended in medicine outpatient department during the period of 2 months from April 2018 to June 2018. Diabetes patients were chosen based on the medical records. Table 1 summarizes the age group distribution of the participants. A pretested questionnaire was prepared into three sections. The first section depicted the sociodemographic profile, including age, gender, occupation and monthly income, the second section comprised lifestyle practices such as dietary practices, including the variety of food, amount of sugar consumed, intake of fruits, type of oil used for cooking, foods and fruits to be avoided, proportion of food in their food plate, consumption of beverages with or without sugar, and awareness of diet chart were included in the questionnaire. In physical activity, questions included the type, duration, and frequency of physical exercise and awareness regarding the brisk walking. In addition, practices such as consumption of smoking and alcohol intake were assessed. The third section included medical history (including duration of diabetes) and comorbid illness such as hypertension, thyroid disorders, and awareness regarding the health education and initiatives of diabetes were assessed. Informed consent was obtained from the study participants. The data collected were recorded in Microsoft Excel and analysed using SPSS version 16.0. The results are presented as proportions and any difference between two proportions in relation to a particular factor was assessed by a Chi-square test (or Fischer exact test if the expected frequency in any cell was $<5$ ) and was considered significant at $p<0.05$.

\section{Results}

The mean age of the research participants was found to be $50 \pm$ 9.64. Among 100 participants, 52 were male and 48 were female

Table 1: Sociodemographic profile of the study population

\begin{tabular}{lll}
\hline & Variables & $N=100(\%)$ \\
\hline Age (years) & $40-49$ & 22 \\
& $50-59$ & 48 \\
Sex & 60 or above & 30 \\
\multirow{4}{*}{ Occupation } & Male & 52 \\
& Female & 48 \\
Education & Heavy worker & 4 \\
\multirow{3}{*}{ Socio-economic status } & Moderate worker & 42 \\
& Sedentary worker & 54 \\
& Literate & 77 \\
& Illiterate & 23 \\
& Upper & 2 \\
& Upper-middle & 3 \\
& Upper-lower & 23 \\
Duration of diabetes & Lower-middle & 48 \\
& Lower & 24 \\
& $1-5$ years & 47 \\
& 5-10 years & 40 \\
& 10-15 years & 9 \\
& 15-20 years & 4 \\
& Chronic kidney disease & 10 \\
& Hypertension & 47 \\
& No comorbidity & 43 \\
\hline
\end{tabular}

patients. An estimated $78 \%$ were above the age of 50 years. Only $71 \%$ of study population were found unaware regarding dietary habits and physical activity. Amidst 100 participants, $47 \%$ and $40 \%$ had the duration of diabetes of $<5$ years and 5-10 years, respectively. The family income was found to be less than Rs. 20,000 in $48 \%$ and Rs. 30,000 among $24 \%$ among the research participants. The socioeconomic status was assessed by Modified Kuppuswamy Classification Socioeconomic Status Scale $2018 .^{12}$ This scale comprises family income, occupation status, and Education of head of the family. The sociodemographic and clinical characteristics are shown in Table 1. Among the research participants, $29 \%$ were alcoholic and $25 \%$ were smokers and all were males. An estimated $12.39 \%$ and $1 \%$ of research participants had hypertension and chronic kidney disease, respectively; $74 \%$ of the research participants were aware of foods to be avoided; $54 \%$ were familiar with the proportion of food required in food plate. Awareness of diet and lifestyle practices among the DM patients are depicted in Table 2. Type of cooking oil used by the diabetic patients is depicted in Figure 1. Physical exercise was regularly done by $57 \%$ of the study population. Figure 2 depicts the types of exercises performed by research participants. Scoring was done for awareness and practice regarding the diet, exercise, and health education programs. It was observed that the median for the awareness's score was 6.5 and the median for practice score was 6 . Awareness score was classified as poor, fair, and good: a score of $0-3=$ poor, $4-7=$ fair, and $8-10=$ good (Fig. 3). There was good awareness score in $29 \%$ of the participants, of which $46 \%$ were females and $54 \%$ were males. Overall, the practice score was categorized as poor, fair, and good: A score of $0-2=$ poor, 3-5 = fair,

Table 2: Awareness of diet, exercise, and other practices among the DM patients

\begin{tabular}{ll}
\hline Awareness items & Responses (\%) \\
\hline Diet & \\
Daily recommended fruits intake & Yes (7) \\
& No (93) \\
Fruits to be avoided & Yes (45) \\
& No (55) \\
Oil to be used & Yes (62) \\
& No (38) \\
Foods to be avoided & Yes (74) \\
& No (26) \\
Proportion of food in a food plate & Yes (54) \\
Diet chart awareness & No (46) \\
Exercise & Yes (58) \\
Aware of brisk walking & No (42) \\
Behavioral characteristics & Yes (57) \\
Smoking habit & No (43) \\
Number of cigarettes & Yes (25) \\
Alcohol consumption & No (75) \\
& One pack/day (36) \\
& Two packs/day (16) \\
& More than two pack/day (48) \\
& Yes (29) \\
& No (71) \\
&
\end{tabular}




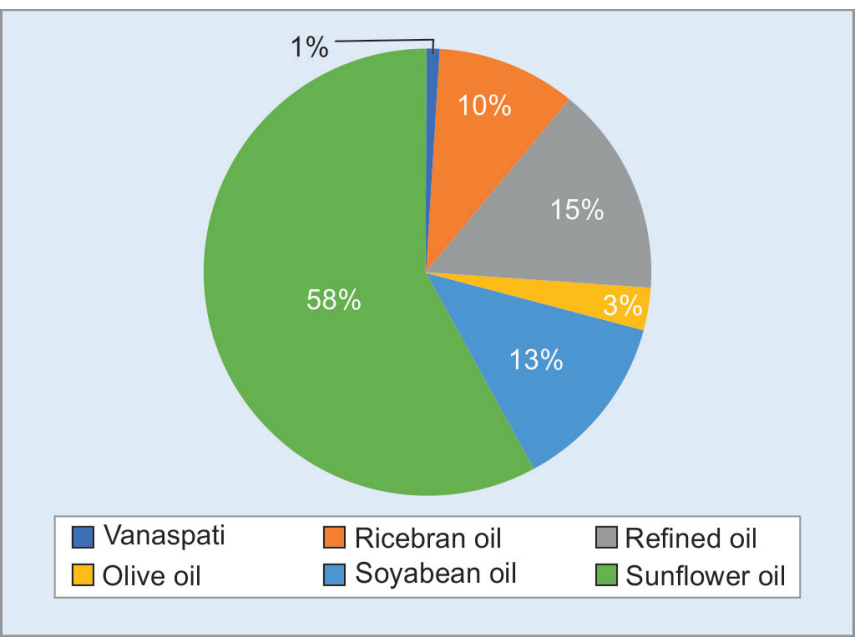

Fig. 1: Types of cooking oil used by diabetes patients

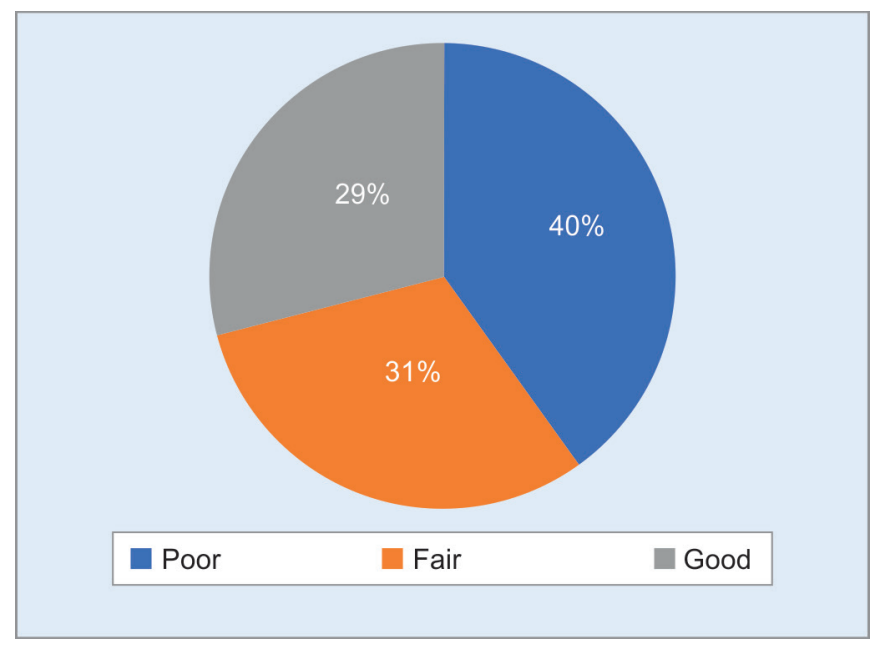

Fig. 3: Overall awareness of diet, physical activity, and health education

and $6-9=$ good. A good practice score was found in $15 \%$ of the research group (Fig. 4).

\section{Discussion}

In our study, more than $61 \%$ of diabetic patients had low socioeconomic status; therefore, they could not pursue the dietary modifications and regular health checkup required by a diabetic patient. Kaiser and Baumann have confirmed similar findings. ${ }^{13}$ Poverty limits the accessibility and affordability of balanced diet, which could have precipitated most of the participants to faulter the lifestyle modification practice score inspite of having a positive approach. Thereby participants from lower socioeconomic status consumed rice in majority of their meals (mostly 3 servings/ day) with limited proportion of proteins and vegetables. The Shanghai Women's Health Study ${ }^{6,14}$ claimed that the increased intake of foods with a high glycemic index (especially white rice) is associated with worsening glycemic control and thereby diabetes status. It was found that with improving socioeconomic status, the lifestyle modifications practice score also increased, which showed a significant association with statistical significance ( $p=$ 0.01 ) Table 3. The study revealed $74 \%$ patients with type 2 diabetes required weight reduction through amalgamation of lifestyle

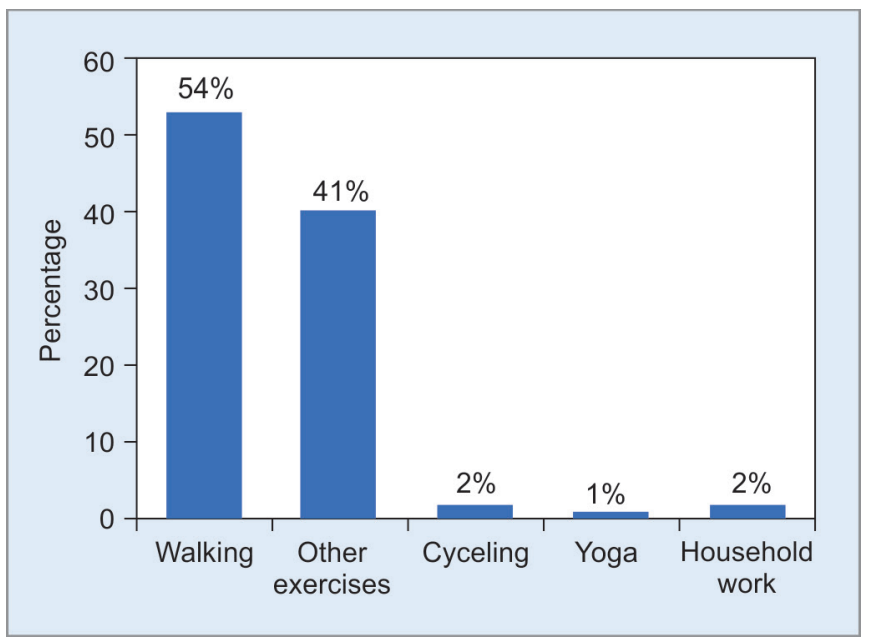

Fig. 2: Types of exercises done by the study group

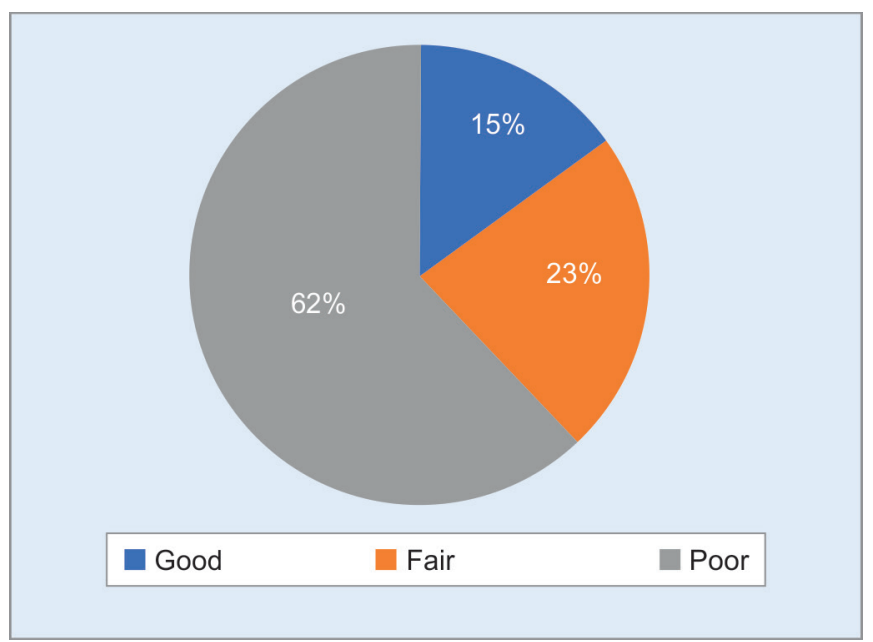

Fig. 4: Practice score of life-style modifications

modifications and the reduction of calorie intake. It is prudent to consume whole grains, vegetables, legumes, diet with high fiber, and low glycemic index food. Diabetic patients must avoid sugar-sweetened beverages and minimize the ingestion of foods with added sugar that have the capacity to replace healthier, more protein and vitamins rich food choices. ${ }^{15,16}$ The American Dietetic Association also highlights that foods if consumed in balance with a suitable portion size in amalgmation with regular physical activity are ideal to maintain the euglycemic status. ${ }^{17}$ As suggested by Evert et al., ${ }^{18}$ nutrition therapy is recommended for type 2 diabetes patients as a crucial factor for the overall management plan. In this research, $25 \%$ of the male diabetic patients were smokers and $29 \%$

Table 3: Correlation of awareness, attitude, and practices among type 2 diabetics

\begin{tabular}{lcc}
\hline Parameters & Pearson's correlation & pvalue \\
\hline $\begin{array}{l}\text { Lifestyle modifications awareness } \\
\text { and attitude }\end{array}$ & 0.14 & 0.01 \\
$\begin{array}{l}\text { Lifestyle modifications awareness } \\
\text { and practice }\end{array}$ & -0.54 & 0.02 \\
$\begin{array}{l}\text { Lifestyle modifications attitude and } \\
\text { practice }\end{array}$ & -0.85 & 0.01 \\
\hline
\end{tabular}


used to consume alcohol. Smoking causes activation of sympathetic nervous system activity, thereby escalating the blood cortisol levels, which leads to visceral adiposity. ${ }^{19}$ Some animal studies have revealed $\beta$-cell dysfunction and increase $\beta$-cell apoptosis with nicotine exposure ${ }^{20}$ and consumption of alcohol among diabetics is linked with chronic pancreatitis, which leads to alcohol-induced liver changes. ${ }^{21}$ Thus smoking and alcohol cessation counseling should also be inculcated as a routine component of diabetes care. Concomitant alcohol and smoking has a concreted effect on the risk of diabetes seen commonly in Indians. Sedentary lifestyle is defined "as sitting or lying down, with low energy expenditure, during waking hours." 22 Only $54 \%$ of diabetics in the study group were involved in sedentary lifestyle, which poses higher risk of diabetes when compared to moderate and heavy workers. It is found that habitual physical exercise can reverse diabetes. ${ }^{23}$ Perseghin et al. proved that moderate intensity exercise of single bout could increase the uptake of glucose by $40 \% .{ }^{23,24}$ Diabetes is also strongly linked with escalated production of reactive oxygen species. ${ }^{23,25}$ Research studies depicted that endurance training uplifts the levels of antioxidant enzymes and antioxidants in cardiac and skeletal muscles, thus shielding from oxidative stress. ${ }^{26}$ The inflammatory markers of endothelial dysfunction such as soluble intracellular and vascular adhesion molecules and granulocyte-macrophage colony-stimulating factor are abridged by exercise. ${ }^{23}$ Physical exercise also ensures nitric oxide availability, thus improving endothelial function. ${ }^{27}$ In this research, yoga was practiced by $1 \%$ of the study population. On the basis of individual preferences, Yoga can be included as a part of mandatory physical activity among diabetics. As $29 \%$ had good lifestyle modification awareness score, $46 \%$ were females and $54 \%$ were males. Overall, lifestyle modification awareness score was found to be high in males compared to females. This may be because of the differences in literacy level, training received, and availability of information on type 2 diabetes. It was also found that with advancement in age, the lifestyle modification awareness score decreases. On the other hand, the lifestyle modification practice score increases with age and duration of the diabetes. When the duration of diabetes increases, the comorbidity also increases, hence the lifestyle modification practice. In this study, we found the risk of comorbidity was about $37 \%$ among those with duration of diabetes for more than 10 years (Table 4).

This study emphasizes the need of rectification of cognizance of dietary and lifestyle modification practices among the diabetics to attain optimum control of diabetes. This accentuates the need for amplifying diabetes cognizance activities in the form of mass campaigns and organized medical nutrition programs in India. The nutritionist could calculate the caloric needs of the individual based on their lifestyle, including physical activity, occupation, and body mass index, and thus suggest low glycemic food choices. Public health policies should be aimed at increasing awareness and uplifting alcohol cessation activities. Psychosocial care should also be mandated to all diabetes, with the goals of improving the quality-of-life. This research is a single-centered study and this study

Table 4: Life-style modifications practice score

\begin{tabular}{ll}
\hline Life-style modifications practice score & $(\%)$ \\
\hline Good & 15 \\
Fair & 23 \\
Poor & 62 \\
\hline
\end{tabular}

did not include the knowledge of glycemic status among diabetic patients, thereby forming the limitations of the study.

\section{Conclusion}

The observations of our study reveal that the awareness and practice of lifestyle modifications among type 2 DM patients attending Tertiary Care Hospital were considerably poor and the dietary restrictions needed for the patients were found to be inadequate. Counseling programs' implementation in diabetes management programs will bring in a drastic impact in providing accelerated medical management of patients with the help of dieticians and doctors of India. Implementation of hospital-based lifestyle intervention program would enhance the knowledge and practice of patients regarding lifestyle modifications among diabetics in various parts of India, thereby improving the morbidity status of diabetes.

\section{References}

1. Kaveeshwar SA, Cornwall J. The current state of diabetes mellitus in India. Australas Med J 2014;7:45-48. DOI: 10.4066/AMJ.2014.1979.

2. Joshi SR, Parikh RM. India-diabetes capital of the world: Now heading towards hypertension. J Assoc Physicians India 2007;55:323-324.

3. Anjana RM, Ali MK, Pradeepa R, et al. The need for obtaining accurate nationwide estimates of diabetes prevalence in India - rationale for a national study on diabetes. Indian J Med Res 2011;133:369-380.

4. American Diabetes Association. Diagnosis and classification of diabetes mellitus. Diabetes Care 2010;33(1):S62-S69. DOI: 10.2337/ dc10-S062.

5. Ranasinghe $P$, Pigera AS, Ishara $M H$, et al. Knowledge and perceptions about diet and physical activity among Sri Lankan adults with diabetes mellitus: a qualitative study. BMC Public Health 2015;15:1160. DOI: 10.1186/s12889-015-2518-3.

6. Hu FB. Globalization of diabetes. Diabetes Care 2011;34:1249-1257. DOI: $10.2337 / \mathrm{dc} 11-0442$.

7. Das SK, Sanyal K, Basu A. Study of urban community survey in India: Growing trend of high prevalence of hypertension in a developing country. Int J Med Sci 2005;2:70-78. DOI: 10.7150/ijms. 2.70 .

8. Colberg SR. Exercise and Type 2 diabetes: The American college of sports medicine and the American diabetes association: Joint position statement. Diabetes Care 2010;33:e147-e167. DOI: 10.2337/ dc10-9990.

9. Colberg SR. Key points from the updated guidelines on exercise and diabetes. Front Endocrinol (Lausanne) 2017;8:33. DOI: 10.3389/ fendo.2017.00033.

10. Hayes L, White $M$, Unwin $N$, et al. Patterns of physical activity and relationship with riskmarkers for cardiovascular disease and diabetes in Indian, Pakistani, Bangladeshi and European adults in a UK population. J Public Health Med 2002;24:170-178. DOI: 10.1093/pubmed/ 24.3.170.

11. Khunti K, Stone MA, Bankart J, et al. Physical activity and sedentary behaviours of south Asian and white European children in inner city secondary schools in the UK. Fam Pract 2007;24:237-244. DOI: 10.1093/fampra/cmm013.

12. Saleem SM. Modified Kuppuswamy scale updated for Year 2018. Paripex Indian J Res March-2018;7(3):217-218.

13. Kaiser BL, Baumann LC. Perspectives on healthy behaviors among low-income Latino and non-Latino adults in two rural counties. Public Health Nurs 2010;27:528-536. DOI: 10.1111/j.1525-1446.2010. 00893.x.

14. Villegas R, Liu S, Gao YT, et al. Prospective study of dietary carbohydrates, glycemic index, glycemic load, and incidence of Type 2 diabetes mellitus in middle-aged Chinese women. Arch Intern Med 2007;167:2310-2316. DOI: 10.1001/archinte.167.21.2310. 
15. Hu FB, Malik VS. Sugar-sweetened beverages and risk of obesity and Type 2 diabetes: Epidemiologic evidence. Physiol Behav 2010;100: 47-54. DOI: 10.1016/j.physbeh.2010.01.036.

16. Hu FB. Resolved: There is sufficient scientific evidence that decreasing sugar-sweetened beverage consumption will reduce the prevalence of obesity and obesity-related diseases. Obes Rev 2013;14:606-619. DOI: $10.1111 /$ obr.12040.

17. Freeland-Graves J, Nitzke S. Position of the American dietetic association: Total diet approach to communication food and nutrition information. J Acad Nutr Dietetics 2007;107:1224-1232. DOI: 10.1016/ j.jada.2007.05.025.

18. Evert AB, Boucher JL, Cypress M, et al. Nutrition therapy recommendations for the management of adults with diabetes. Diabetes Care 2013;36:3821-3842. DOI: 10.2337/dc13-2042.

19. Tweed JO, Hsia SH, Lutfy K, et al. The endocrine effects of nicotine and cigarette smoke. Trends Endocrinol Metab 2012;23:334-342. DOI: 10.1016/j.tem.2012.03.006.

20. Xie XT, Liu Q, Wu J, et al. Impact of cigarette smoking in Type 2 diabetes development. Acta Pharmacol Sin 2009;30:784-787. DOI: 10.1038/aps.2009.49.

21. Kim SJ, Kim DJ. Alcoholism and diabetes mellitus. Diabetes Metab J 2012;36:108-115. DOI: 10.4093/dmj.2012.36.2.108.
22. Owen N, Sparling PB, Healy GN, et al. Sedentary behavior: Emerging evidence for a new health risk. Mayo Clin Proc 2010;85:1138-1141. DOI: $10.4065 / \mathrm{mcp} .2010 .0444$.

23. Venkatasamy VV, Pericherla S, Manthuruthil S, et al. Effect of physical activity on insulin resistance, inflammation and oxidative stress in diabetes mellitus. J Clin Diagn Res 2013;7:1764-1766. DOI: 10.7860/ JCDR/2013/6518.3306.

24. Perseghin G, Price TB, Petersen KF, et al. Increased glucose transportphosphorylation and muscle glycogen synthesis after exercise training in insulin-resistant subjects. N Engl J Med 1996;335: 1357-1362. DOI: 10.1056/NEJM199610313351804.

25. Ceriello A, Motz E. Is oxidative stress the pathogenic mechanism underlying insulin resistance, diabetes, and cardiovascular disease? The common soil hypothesis revisited. Arterioscler Thromb Vasc Biol 2004;24:816-823. DOI: 10.1161/01.ATV.0000122852.22604.78.

26. Leeuwenburgh C, Fiebig R, Chandwaney R, et al. Aging and exercise training in skeletal muscle: Responses of glutathione and antioxidant enzyme systems. Am J Physiol 1994;267:R439-R445. DOI: 10.1152/ ajpregu.1994.267.2.R439.

27. Taddei S, Galetta F, Virdis A, et al. Physical activity prevents agerelated impairment in nitric oxide availability in elderly athletes. Circulation 2000;101:2896-2901. DOI: 10.1161/01.CIR.101.25.2896. 